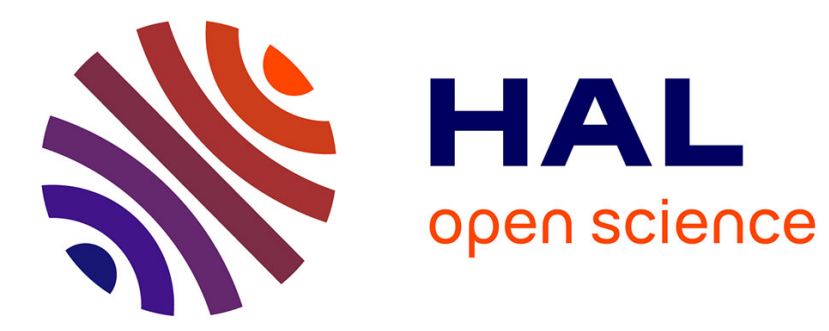

\title{
Bayesian Inference of a Human Bone and Biomaterials Using Ultrasonic Transmitted Signals
}

\author{
Rémi Roncen, Zine El Abiddine Fellah, Estelle Piot, Erick Ogam
}

\section{To cite this version:}

Rémi Roncen, Zine El Abiddine Fellah, Estelle Piot, Erick Ogam. Bayesian Inference of a Human Bone and Biomaterials Using Ultrasonic Transmitted Signals. Journal of the Acoustical Society of America, 2019, 146 (3), pp.1629. 10.1121/1.5125263 . hal-03036901

\section{HAL Id: hal-03036901 https://hal.science/hal-03036901}

Submitted on 2 Dec 2020

HAL is a multi-disciplinary open access archive for the deposit and dissemination of scientific research documents, whether they are published or not. The documents may come from teaching and research institutions in France or abroad, or from public or private research centers.
L'archive ouverte pluridisciplinaire HAL, est destinée au dépôt et à la diffusion de documents scientifiques de niveau recherche, publiés ou non, émanant des établissements d'enseignement et de recherche français ou étrangers, des laboratoires publics ou privés. 


\title{
Bayesian Inference of a Human Bone and Biomaterials Using
}

\section{Ultrasonic Transmitted Signals}

\author{
R. Roncen ${ }^{* 1}$, Z.E.A. Fellah ${ }^{2}$, E. Piot $^{1}$ and E. Ogam ${ }^{2}$ \\ ${ }^{1}$ ONERA / Département Multi-Physique pour l'Énergétique, Université de Toulouse, F-31055, Toulouse, France \\ ${ }^{2}$ Laboratoire de Mécanique et d'Acoustique, Centre National de la Recherche Scientifique, Unité Mixte de \\ Recherche 7031, Aix-Marseille Université, Centrale Marseille, F-13402 Marseille Cedex 20, France
}

\begin{abstract}
Ultrasonic techniques could be good candidates to aid the assessment of osteoporosis detection, due to their non-intrusiveness and low cost. While earlier studies made use of the measured ultrasonic phase velocity and attenuation inside the bone, very few have considered an inverse identification of both the intrinsic pore microstructure and the mechanical properties of the bone, based on Biot's model. The main purpose of this work is to present an in vitro methodology for bone identification, adopting a statistical Bayesian inference technique using ultrasonic transmitted signals, which allows the retrieval of the identified parameters and their uncertainty. In addition to the bone density, Young's modulus and Poisson's ratio, the bone pore microstructure parameters (porosity, tortuosity and viscous length) are identified. These additional microstructural terms could improve our knowledge on the correlations between bone microstructure and bone diseases, since they provide more information on the trabecular structure. In general, the exact properties of the saturating fluid are unknown (bone marrow and blood in the case of bone study) so in this work, the fluid properties (water) are identified during the inference as a proof of concept.
\end{abstract}

\section{Introduction}

Osteoporosis is a metabolic bone disorder that affects the bone density and microstructure [1], progressively reducing bone quality and increasing the risk of fractures. The pathology is particularly prevalent in

\footnotetext{
*Corresponding author: remi.roncen@onera.fr
} 
post-menopause women, due to the loss of estrogen, which reduces the rate of bone matrix formation (osteoblasts) compared with that of bone removal (osteoclasts). Osteoporosis detection is routinely conducted via X-Ray absorptiometry methods, which evaluate the bone mass density (BMD). It has also been shown that astronauts suffer from bone loss while in space [2, 3] due to the micro gravity environment (up to a $10 \%$ loss over a 6 month period). While the cause of bone loss in space is still not fully comprehended, it has been suggested that changes in bones are different from age-related bone loss. Space programs have thus been looking at new ways to extend bone monitoring during the mission, beyond that of classical dual-energy $\mathrm{X}$-Ray technologies [2, 3].

To avoid the invasive treatments that current radiographic or neutron activation technologies require for bone analysis, quantitative ultrasound assessments have been considered for more than 30 years now [413. (for an osteoporosis-focused review, see Refs. [14, 15]). In the seminal paper by Langton et al. [4], the broadband ultrasonic attenuation (BUA) of in vivo and in vitro bovine cancellous spongy bones was measured, showing its strong correlation to the BMD. However, BMD alone does not fully account for fracture risks: for a given BMD, the risk of fracture still increases with age, and there is an overlap in bone density between women with and without fracture [15]. Overall, BMD is thought to explain around $70 \%$ of bone strength, the rest being attributed to bone microstructure or remodeling states [14].

A first refinement to the BUA measurement was achieved by measuring the ultrasonic wave speed [4, 5, 10] of a wave traveling through the bone. It was shown that bones suffering from osteoporotic disorders would have a different wave behavior compared with that of a non-osteoporotic bone. Recognizing that cancellous bones are essentially porous media filled with a fluid phase (the tissue, or marrow, can be considered as a visco-elastic fluid), Hosokawa \& Otani [10] used Biot's theory [16] to explain the appearance of a slow wave and a fast wave, and their correlations to bone density. Bone volume fraction (or equivalently, the porosity) was shown to be a relevant microstructure indicator due to its influence on both waves, and an important parameter to characterize.

Biot's theory has received a lot of attention for its application to ultrasound propagation through bone [5, 12, 17 21] (for a cancellous bone focused review, see [22]), with the predominant concern being the inability of Biot's theory to accurately reproduce BUA measurements (under-estimation of the BUA). Two non-Biot attenuation mechanisms were then proposed to account for the observed discrepancies: a local squirt-flow mechanism in micro-cracks (see [23] for an application to geophysics; has not been studied in the specific case of bones to the best of the authors knowledge), or a possible roughness of the pores (see [24] for 
an application to sandstone). However, McKelvie \& Palmer [5] showed that when taking into account a frequency dependent tortuosity, the BUA match between experimental measurements and Biot's theory could be improved. This observation led to the taking into account of additional visco-inertial frequency dependent dissipation mechanisms, as predicted by the semi-phenomenological model of Johnson, Koplik and Dashen (hereafter JKD [25]), which relates the intrinsic properties of the microstructure to the wave propagation within the fluid. The updated theory, coined Biot-JKD, was then used to try and obtain more information on the bone microstructure, now considered explicitly within the model [26, 27. Fellah et al. [12] calculated the expressions of the reflection and transmission coefficients of a bone sample in the frequency domain, and showed the sensitivity of the different model parameters (porosity, tortuosity, Young's modulus, etc) on time-domain transmitted signals, and on the fast and slow waves. Alternatively, the analytical expressions of the reflection and transmission operators were derived in the time domain, using fractional derivative calculus [28, with application to human cancellous bone. Sebaa et al. [29] used time-domain ultrasonic transmitted signals at normal incidence on water-filled human cancellous bone samples, to solve an inverse problem using the Biot-JKD model. A total of five parameters were deduced: the porosity, the tortuosity, the viscous characteristic length, the Young's modulus and the Poisson's ratio of the solid frame. The inverse problem was claimed to be well posed, meaning that a unique solution exists and that the direct problem is well-conditioned. However, only a local analysis was performed on a point of minimum obtained through a least-mean square fit of the experimental signals. It appeared clear at the beginning of the present study that inverse problems such as this one are not as well posed as claimed initially. This difficulty was also observed in Refs. [30-32]. Combining a wave based method with measurements performed in a Kundt tube, Vanhuyse et al. [33] performed the inverse problem to retrieve all the parameters of the Biot-JCA model (Johnson-Champoux-Allard [25, 34]), an extension of the Biot-JKD model that takes into account thermal dissipation in the fluid phase. A global optimization method was applied for audible signals obtained in air, showing the capability of such a method to retrieve the model parameters, provided that a clamped condition be assumed for the sample within the tube. One of the main difficulties when measuring intrinsic properties of a poroelastic material such as a bone, is ensuring that the experimental conditions (sample holder) do not interfere with the sample mechanical properties. In addition, the audible frequency regime usually resides at intermediary frequencies, between the low (viscous isothermal fluid) and high (inertial adiabatic fluid) regimes. Since the JKD model is essentially a fit between the behaviors obtained at the two limits of the frequency spectrum [25], it is adequate to look for intrinsic parameters in the frequency domain 
where they have been defined. As we are interested in the tortuosity and characteristic viscous length of a bone (which we believe could prove to be indicators of interest in osteoporosis monitoring, as strongly hinted in Ref. [5] where the pore size, porosity and permeability of the bone are considered), both defined in the high frequency inertial regime, it is understandable why the present study focuses on ultrasonic signals.

While the previous studies focused on a deterministic approach to inverse identification, i.e., a minimization of a least mean square difference between experimental and numerical signals, no account on uncertainties was given. Using frequency-domain signals obtained in an impedance tube, Chazot et al. [35] followed a Bayesian inference approach to measure the Biot-JCA parameters and associated uncertainties, caused by the inherent uncertainties in the experiment and the modeling. They used an evolutionary Markov-Chain Monte-Carlo procedure to calculate the posterior probability density of each parameter, showing the presence of multi-modal densities. Bayesian inference, as we shall see in the present work, is a learning rule based on Bayes's theorem: the information one has on a parameter is updated through the observation of a new quantity. More recently, a Bayesian inference technique has been used on synthetic noisy signals from both the reflection and transmission coefficients of a porous sample in the ultrasonic domain, showing the capability of Bayesian inference to inverse the value of each parameter of the Biot-JKD model, and the influence of the angle of incidence on the inference [36. Alternatively, different studies have been conducted, using a Bayesian inference process, to retrieve quantities such as the wave speed of attenuation of both fast and slow waves, even in the case when they are difficult to separate [37, 38. While these properties are strongly correlated to the porous microstructure, they are not intrinsic in that they depend on the ambient fluid.

Ultrasonic transmitted waves are considered in this work at almost normal incidence on porous samples immersed in water. A Bayesian inference approach is then performed to update our state of belief on the Biot-JKD parameters, which consist of the classical Biot parameters (density, Young's modulus, Poisson's ratio etc) and the intrinsic microstructure properties (porosity, tortuosity, viscous length). In addition, the exact value of the incidence angle is assumed unknown, due to the slightly slanted faces of the bone sample after its preparation, and of the sample holder precision. While the properties of the ambient fluid (water) are relatively well known, we consider these properties to be part of the inference problem in an effort to tackle the possible future challenges of in vivo ultrasonic measurement, where the fluid properties would be uncertain. The study is organized as follows.

The acoustical Biot-JKD modeling is recalled in Section III The experimental configuration used in this 
work is given in Section [II] where a sensitivity analysis is performed to see the influence of the Biot-JKD parameters on synthetic transmitted signals mimicking the experiment. Then, the statistical Bayesian inference problem is introduced in Section IV] The inference method is then applied on experimental measurements on 2 ceramic biomaterial porous and a bone sample from a femoral head, in Section $\mathrm{V}$. A conclusion is drawn in Section VI

\section{Acoustical model}

A bone, be it cancellous or cortical, can be seen as a porous material in which two phases interact with each other. The fluid phase, composed of blood and marrow, is a viscoelastic fluid. The solid phase, made of calcified tissue, has a complex microstructure that pertains to its physical strength. Due to the two phases having a density of the same order of magnitude (i.e $\approx 1 \cdot 10^{3} \mathrm{~kg} / \mathrm{m}^{3}$ for the fluid phase and $\approx 2 \cdot 10^{3} \mathrm{~kg} / \mathrm{m}^{3}$ for the solid phase), coupling effects between phases can be important. This is one of the main differences with sound absorbing porous media, which are mostly considered in air where the coupling effect is less pronounced.

Biot's initial theory describes the equations of motion of the two phases [16, involving 6 unknowns, which are the displacement fields of the two phases. In this work, we use Biot's alternative formulation [39], while taking into account the additional visco-inertial dissipation that occurs within the pores, as developed by Johnson et al. 25]. In this regard, we mostly follow Niskanen et al. [32] in the presentation of the modeling strategy, using the same notations (except when mentioned otherwise).

Equations are written in the frequency domain, where the field quantities are the Fourier transform coefficients of their time domain counterpart ( $e^{+\mathrm{j} \omega t}$ convention), homogenized from a representative elementary volume (REV), small compared with the wave length considered, large compared with the pore size. Biot's coupling equations are given as

$$
\begin{gathered}
\omega^{2} \rho_{f} \boldsymbol{w}+\omega^{2} \rho \boldsymbol{u}=-\Delta \cdot \boldsymbol{\sigma}, \\
\omega^{2} \rho_{f} \boldsymbol{u}+\omega^{2} \tilde{\rho}_{\mathrm{eq}} \boldsymbol{w}=\Delta \cdot p,
\end{gathered}
$$

where $p$ is the pressure field, $\boldsymbol{\sigma}$ is the total stress tensor, $\boldsymbol{u}$ is the solid phase displacement field, $\boldsymbol{w}$ is the relative displacement field between the phases: $\boldsymbol{w}=\phi(\boldsymbol{U}-\boldsymbol{u})$ with $\boldsymbol{U}$ the fluid phase displacement field and $\phi$ the porosity. Note that the coupling between the solid and fluid phases is of a volume nature, the phases being considered superposed in both time and space [40, Chap. 6]. The angular frequency writes $\omega=2 \pi f$, 
with $f$ the frequency in $\mathrm{Hz} ; \rho_{f}$ is the ambient fluid density and $\rho=(1-\phi) \rho_{s}+\phi \rho_{f}$ is the density of the bulk medium, with $\rho_{s}$ the density of the calcified tissue. The equivalent density of the fluid phase $\tilde{\rho}_{\text {eq }}$ is a complex frequency dependent quantity that takes into account visco-inertial effects within the fluid phase inside the pores. The equivalent density reads

$$
\tilde{\rho}_{\mathrm{eq}}=\frac{\rho_{f}}{\phi} \tilde{\alpha}(\omega)
$$

with $\tilde{\alpha}(\omega)$ the dynamic tortuosity, defined in the high frequency limit, where the viscous effects are concentrated in a small volume near the surface of the frame and are negligible compared with inertial effects within the pores, as

$$
\tilde{\alpha}(\omega)=\alpha_{\infty}\left(1+\frac{2}{\Lambda} \delta_{v}+\mathcal{O}\left(\delta_{v}^{2}\right)\right)
$$

where $\alpha_{\infty}$ is the high frequency limit of the dynamic tortuosity (hereafter denoted simply "tortuosity"), a dimensionless ratio related to the disorder of the microstructure [25]; $\Lambda$ is the characteristic viscous length [25], close to the value of the smallest hydraulic radius of the pore connections, where the slow wave propagates and where viscous effects are dominant. The viscous boundary layer for viscous effects $\delta_{v}$ is defined as

$$
\delta_{v}=\sqrt{\frac{\mu_{f}}{\mathrm{j} \omega \rho_{f}}}
$$

where $\mu_{f}$ is the intra-pore fluid dynamic viscosity. The high-frequency regime is attained when $\delta_{v} \ll r_{p}$, with $r_{p}$ the typical size of a pore. In the present applications, where $\mu_{f} \approx 1 \cdot 10^{-3} \mathrm{~kg} \cdot \mathrm{m}^{-1} \cdot \mathrm{s}^{-1}, \rho_{f} \approx 1 \cdot 10^{3} \mathrm{~kg} \cdot \mathrm{m}^{-3}$ and $r_{p}$ could be as low as $r_{p} \approx 5 \mu \mathrm{m}$ at a constriction, the lower bound for the frequency to be in the high frequency regime is $f_{v} \gg 6.4 \mathrm{kHz}$. Since we consider signals in the ultrasonic range $(f>500 \mathrm{kHz})$, we comply with the requirements for the hypothesis of a high frequency regime, while keeping the wave-length large compared with the pore size $\left(\lambda_{v}=c_{f} / f \approx 1500 / 5 \cdot 10^{5}=3 \mathrm{~mm}\right)$. We note that this further assumption could prove false in some highly porous cancellous bone with pore diameters $\approx 1 \mathrm{~mm}$ [4], which could lead to the need for an additional modeling effort regarding the presence of Rayleigh scattering effects [42]. This limitation could be avoided by using a signal of a lower frequency content.

The presence of coupling between the fluid and solid phases in Eq. 1 is evidenced by the relationship between the total stress tensor $\boldsymbol{\sigma}$ and the pressure field $p$, which reads, for an isotropic porous material (at the wave-length scale), as

$$
\begin{aligned}
& \boldsymbol{\sigma}=2 N \boldsymbol{\epsilon}+\left(\lambda_{c} \xi-\alpha_{B} M \zeta\right) \mathbb{I}, \\
& p=M\left(\zeta-\alpha_{B} \xi\right)
\end{aligned}
$$


where $N$ is the shear modulus, $\boldsymbol{\epsilon}=\frac{1}{2}\left(\nabla \boldsymbol{u}+(\nabla \boldsymbol{u})^{T}\right)$ is the strain tensor, II is the identity matrix, $\xi=\nabla \cdot \boldsymbol{u}$ ( $\theta$ in Ref. [32] ) and $\zeta=-\nabla \cdot \boldsymbol{w}$. The Biot-Willis coefficient $\alpha_{B}(\alpha$ in Ref. [43, Eq. 28]) writes, after simplifications,

$$
\alpha_{B}=1-\frac{K_{b}}{K_{s}}
$$

In the previous equation, $K_{b}$ is the bulk modulus of the porous frame, while $K_{s}$ is that of the solid constituent of the material. The shear modulus $N$, the Young's modulus and Poisson's ratio of the solid $E_{s}, \nu_{s}$ and the bulk skeletal frame $E_{b}, \nu_{b}$ are related to the bulk moduli by the expressions

$$
K_{s}=\frac{E_{s}}{3\left(1-2 \nu_{s}\right)}, \quad K_{b}=\frac{E_{b}}{3\left(1-2 \nu_{b}\right)}, \quad N=\frac{E_{b}}{2\left(1+\nu_{b}\right)} .
$$

We note that the parameters $E_{s}$ and $\nu_{s}$ are non identifiable. They pertain to the definition of a single parameter, $K_{s}$, so there is not enough information to separate these two values. For this reason, the parameter that is inferred in this work is $K_{s}$. The problem does not arise for $E_{b}$ and $\nu_{b}$, since they are related to both $K_{b}$ and $N$.

In Eq. $5 \lambda_{c}$ is an elastic parameter defined as

$$
\lambda_{c}=\lambda+\alpha_{B}^{2} M
$$

where $\lambda$ is the first Lamé's coefficient of the elastic frame, and

$$
M=\left[\alpha_{B}+\left(\frac{K_{s}}{K_{f}}-1\right) \phi\right]^{-1} K_{s}
$$

with $K_{f}$ the bulk modulus of the intra-pore fluid (thermal effects are neglected in water).

The analytical calculation in the frequency domain of the transmission coefficient $\mathcal{T}(\omega)$ of a bone sample is done by solving the system (1] and associated boundary conditions [40, Chap. 6]. We follow, as was done in Ref. [32], the state vector formalism that makes it possible to write the motion equations as an ordinary differential equation, coupled to a global matrix approach to a solution for the Biot equations [44, 45]. Physical fields are expressed as a function of wave amplitudes, thus naturally introducing the transmission and reflection coefficients in the matrix representation. These coefficients are then extracted after the solving of a linear system, conveniently expressed with a sparse matrix easy to inverse at all frequencies simultaneously. The incident $\left(p_{i}\right)$ and transmitted $\left(p_{t}\right)$ pressure fields are then related to the transmission scattering operator $\tilde{\mathcal{T}}(t)$ via the convolution in the time domain

$$
p_{t}(x, t)=\int_{0}^{t} \tilde{\mathcal{T}}(\tau) p_{i}(t-\tau) \mathrm{d} \tau,
$$


where the incident wave is retrieved by phase-shifting the reference signal $p_{\text {ref }}$ as

$$
p_{i}(t)=p_{\text {ref }}\left(t-\frac{x-L}{c_{f}}\right),
$$

with $L$ the material thickness. The reference pressure signal is measured in the absence of the porous sample, but is not an incident signal. In practice, instead of doing the convolution in Eq. 10, the incident pressure signal is first Fourier transformed, multiplied by the transmission coefficient in the frequency domain, and the product is inverse Fourier transformed as

$$
p_{t}(x, t)=\operatorname{iFFT}\left[\mathcal{T}(\omega) \hat{p}_{i}(\omega)\right] .
$$

\section{Influence of model parameters on the measured transmitted signals}

In this section, the experimental apparatus used to measure transmitted waves through the bone sample is first recalled (it is identical to that in Ref. [29]). Then, a numerical sensitivity analysis is conducted to evaluate the influence of each of the model parameters on the transmitted waves.

\section{A Experimental apparatus}

Experiments are performed in water using two Panametrics A 303S (resp. V389) plane piezoelectric transducers of diameter $1 \mathrm{~cm}$, with a $500 \mathrm{kHz}$ central frequency (resp. $1 \mathrm{MHz}$ ). $400 \mathrm{~V}$ pulses are provided by a 5058PR Panametrics pulser/receiver, amplified to $90 \mathrm{~dB}$, filtered above $10 \mathrm{MHz}$ and averaged over 1000 iterations. A schematic of the experiment is shown in Fig. 1. The size of the ultrasound beam is very small compared with the size of the specimens. The liquid initially in the pore space (blood and marrow) is removed from the bone sample and substituted by water. The bone samples, machined from the cancellous parts of a femoral head and neck, are constantly immersed in liquid to prevent them from drying, which can alter their properties [46]. Due to possible manufacturing defects and positioning uncertainties, the angle of incidence of the pressure wave $\theta$ (displayed in Fig. 1) is taken as an additional unknown.

\section{B Sensitivity analysis}

A synthetically generated numerical incident pressure is considered. The transmitted pressure associated with the transmission through a certain material is then obtained using Eq. 12 , thus mimicking the experiment 


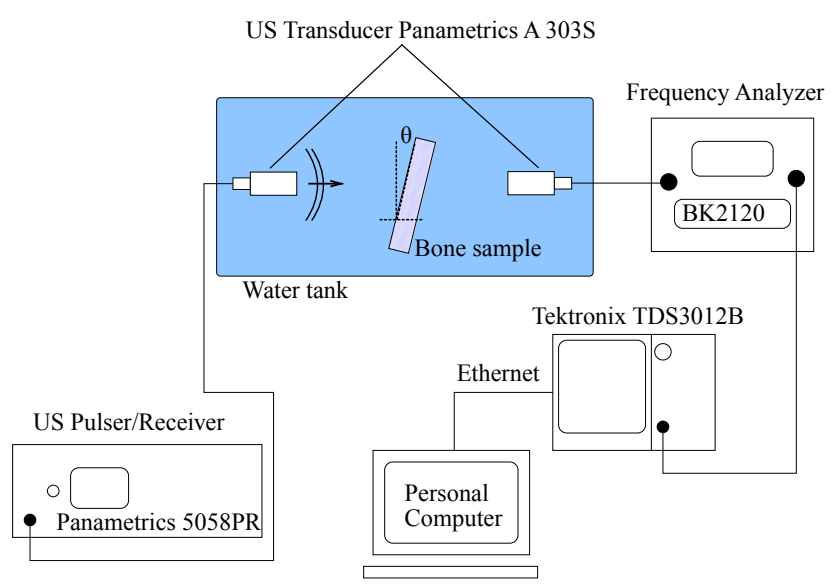

Figure 1: Experimental setup for ultrasonic measurements

described in Sec. A. The influence of all the parameters intervening in the Biot-JKD model of Sec. II is studied, by varying their reference values, other things equal, in the same manner as was done in Ref. [12]. Reference properties that are close to those of bone materials are considered, while ensuring the clear display of both fast and slow waves, at normal incidence only. Reference properties are displayed in Table 1 and the reference transmitted time-domain signal is shown in Fig. 2, where the slow and fast waves are represented when water is considered within the pores.

Note that $\xi_{b}\left(\right.$ resp. $\left.\xi_{s}\right)$ has been introduced in Table 1 as a parameter equal to the ratio of the imaginary part to the real part of the Young modulus of the bulk skeletal frame $E_{b}$ (resp. $E_{s}$ ), to allow for dissipation caused by the frame. 
Table 1: Summary of the reference properties

\begin{tabular}{llc}
\hline \hline Parameter & Symbol & Value \\
\hline Porosity & $\phi$ & 0.75 \\
Tortuosity & $\alpha_{\infty}$ & 1.05 \\
Viscous length & $\Lambda(\mu \mathrm{m})$ & 10.0 \\
Bulk modulus & $K_{s}(\mathrm{GPa})$ & 10.8 \\
Imaginary ratio & $\xi_{s}$ & 0.0 \\
Young modulus & $E_{b}(\mathrm{GPa})$ & 4.5 \\
Imaginary ratio & $\xi_{b}$ & 0.0 \\
Poisson ratio & $\nu_{b}$ & 0.28 \\
Solid density & $\rho_{s}\left(\mathrm{~g} / \mathrm{cm}^{3}\right)$ & 2.0 \\
Fluid bulk modulus & $K_{f}(\mathrm{GPa})$ & 2.0 \\
Fluid viscosity & $\mu_{f}\left(\mathrm{~kg} \cdot \mathrm{m}^{-1} \cdot \mathrm{s}^{-1}\right)$ & $1.0 \cdot 10^{-3}$ \\
Fluid density & $\rho_{f}\left(\mathrm{~g} / \mathrm{cm}^{3}\right)$ & 1.0 \\
Sample thickness & $L(\mathrm{~mm})$ & 20 \\
\hline \hline
\end{tabular}

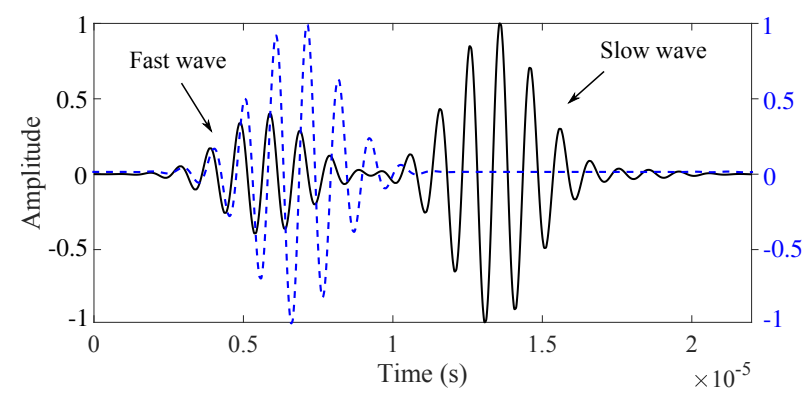

Figure 2: Reference transmitted ultrasonic signal with an air filled bone (straight black line) and with a viscous fluid mimicking marrow (dashed blue line). Incident signal has a Gaussian spectrum centered at $1 \mathrm{MHz}$ with a $100 \mathrm{kHz}$ standard deviation. Signals are normalized by their respective maximum amplitude.

The 12 parameters in Table 1 are now varied. For each parameter variation, the difference in fast and slow wave speeds $\left(c_{\text {fast }}, c_{\text {slow }}\right)$ and amplitudes $\left(A_{\text {fast }}, A_{\text {slow }}\right)$ is given in Table 2 . While these four quantities do not represent the full content of a transmitted ultrasonic signal, they are representative enough so as to give an idea of the influence of the model parameters. 
Table 2: Influence of parameter variation on the fast and slow wave speed and amplitude (in \%), with respect to reference values of Table 1

\begin{tabular}{ccccc}
\hline \hline Parameter & $\Delta_{\%} c_{\text {fast }}$ & $\Delta_{\%} c_{\text {slow }}$ & $\Delta_{\%} A_{\text {fast }}$ & $\Delta_{\%} A_{\text {slow }}$ \\
\hline$\phi+20 \%$ & +41 & +1 & -85 & +45 \\
$\alpha_{\infty}+20 \%$ & -8 & -13 & -181 & -43 \\
$\Lambda+20 \%$ & +0.2 & 0.4 & +13 & +51 \\
$K_{s}+20 \%$ & 0 & -1 & +14 & -12 \\
$\xi_{s}+0.05$ & 0 & -0.3 & -0.2 & +59 \\
$E_{b}+20 \%$ & +10 & 0.8 & -10 & +16 \\
$\xi_{b}+0.05$ & 0 & 0 & -85 & -31 \\
$\nu_{b}+20 \%$ & +9 & +1 & -18 & +28 \\
$\rho_{s}+20 \%$ & -8 & 0 & +4 & +1 \\
$K_{f}+20 \%$ & +3 & +11 & +8 & +20 \\
$\mu_{f}+20 \%$ & -0.7 & -0.3 & -6 & -20 \\
$\rho_{f}+20 \%$ & -5 & -15 & -6 & -20 \\
$L+20 \%$ & -22 & -38 & -21 & -38 \\
\hline \hline
\end{tabular}

All parameters seem to display a certain amount of sensitivity to the ultrasonic transmitted signal, and should thus be considered during the inversion procedure. As expected, parameters associated to the Biot model have a strong influence on the fast wave, while parameters associated to the pore microstructure have a strong influence on the slow wave. However, due to the coupling between the solid and fluid phases in the Biot model, a strong influence of the Biot parameters can be seen on the slow wave (see for instance $\xi_{s}$ ). The same observation holds for the sensitivity of microstructure parameters (i.e. $\alpha_{\infty}$ ) on the fast wave.

When a fluid of higher viscosity is considered $\left(\mu_{f}=10^{-1} \mathrm{~kg} \cdot \mathrm{m}^{-1} \cdot \mathrm{s}^{-1}\right)$, the reference signal displayed in dashed blue in Fig. 2 only shows the presence of the fast wave: the slow wave is much more attenuated in this case. The transmitted signals of Fig. 2 are both normalized by their respective maximum amplitude. In practice, the signal transmitted in the higher viscosity fluid has a maximum that is $\approx 3.4$ times lower than the maximum of the signal transmitted in water. It is thus expected that for in vivo measurements where the bone is filled with marrow (much more viscous than water), the slow wave might prove more difficult to 
detect than in the case of water filled pores. However, Biot theory is still valid even when the slow wave is not visible.

\section{Identification method: Bayesian inference}

In this section, the principal elements of Bayesian inference are reviewed and applied to the calibration of the Biot-JKD model recalled in Sec. II] A numerical strategy is then presented (see Ref. [4] for an application of the same strategy to ultrasonic transmitted waves in rigid porous media filled with air).

\section{A Bayes theorem}

In the context of statistical inverse problems, the Bayesian inference framework consists in recasting the model parameters of interest as random variables associated with probability densities encompassing the information one has on the parameters [48, Chap. 8]. A new experimental data $v^{\text {obs }}$ is observed (here a signal transmitted through the bone sample), and this information updates our state of knowledge. $v^{\text {obs }}$ is the realization of a multi-variate random variable $\Upsilon^{\text {obs }}$. The degree of knowledge about the true value of the parameters $Q=\left(\phi, \alpha_{\infty}, \ldots\right)$, with realizations $q$, is represented by the marginal posterior density $\pi\left(q \mid v^{\text {obs }}\right)$. This new quantity of interest is written, using Bayes theorem,

$$
\pi\left(q \mid v^{\mathrm{obs}}\right)=\frac{\pi\left(v^{\mathrm{obs}} \mid q\right) \pi_{0}(q)}{\pi\left(v^{\mathrm{obs}}\right)}
$$

In the previous equation, $L(q) \equiv \pi\left(v^{\text {obs }} \mid q\right)$ is the likelihood function, representing the probability that the experience be observed, given a particular set of model parameters; $\pi_{0}(q)$ is the prior probability, representing all the information obtained on $q$ prior to the new observation (i.e, earlier measurements on the sample, or general knowledge of some of the bone properties such as its mineral density). Finally, $\pi\left(v^{\text {obs }}\right)$ is a scaling constant not calculated in practice. Details on the likelihood and prior modeling are given hereafter.

\section{A.1 Likelihood}

The physical correlations between the parameters of interest and the experimental data being observed are predicted by a deterministic forward model (Biot-JKD model of Sec. II ) and a deterministic operator $f$ defined as

$$
f_{i}(q)=\hat{p}_{t}\left(t_{i}\right), \quad i=1, \ldots, n
$$

where $\hat{p}_{t}\left(t_{i}\right)$ is a numerical transmitted pressure signal, evaluated using Eq. 12 at time step $t_{i}$. 
Due to both the observation and the modeling being corrupted by uncertainties, it is legitimate to consider that the measurement $\Upsilon^{\text {obs }}$ is related to the deterministic operator by a relationship of the type

$$
\Upsilon_{i}^{\text {obs }}=f_{i}(q)+\varsigma_{j}, \quad i=1, \ldots, n
$$

where $\varsigma_{j}$ is a random variable representing the averaged measurement errors at time steps belonging to zone $j$ (see Fig. 3). In the case where the measurement errors are independent and identically distributed and $\varsigma_{j} \sim \mathcal{N}\left(0, \sigma_{j}^{2}\right)$, then

$$
\Upsilon_{i}^{\mathrm{obs}} \sim \mathcal{N}\left(f_{i}(q), \sigma_{j}^{2}\right)
$$

which leads to

$$
\pi\left(v_{i}^{\mathrm{obs}} \mid q\right)=\frac{1}{\sqrt{2 \pi \sigma_{j}^{2}}} e^{-\left[v_{i}^{\mathrm{obs}}-f_{i}(q)\right]^{2} / 2 \sigma_{j}^{2}} .
$$

When considering all the measurement points (at different time steps) as independent and uncorrelated, the likelihood becomes

$$
\pi\left(v^{\mathrm{obs}} \mid q\right)=\prod_{i=1}^{n} \pi\left(v_{i}^{\mathrm{obs}} \mid q\right)
$$

The additional parameters $\varsigma_{j}$ are unknown and should be identified along with the parameters of interest. However, due to the large number of model parameters and the possibility of over-fitting, it was deemed more conservative to fix the values of $\varsigma_{j}$ to represent a typical spread obtained depending on the ultrasonic beam position along the bone sample, due to strong spatial inhomogeneities. Different zones of the transmitted signal were considered, each associated with a particular averaged value of $\varsigma_{j}$, as represented in Fig. 3 for the reference signal, where four different zones were considered. Had we considered only one single $\varsigma_{j}$ (as in our recent work [47]), certain parts of the signal would have been too constrained, while other parts of the signal would have been too unconstrained, resulting in a poor fit between numerical and experimental signals. The values of the $\varsigma_{j}$ were adjusted manually in each zone prior to the identification, to correspond to our knowledge on the typical measurement spread observed: in zones 1 and 4 , the $\varsigma_{1}$ and $\varsigma_{4}$ value is set to $0.5 \%$ of the maximum signal amplitude, while $\varsigma_{2}$ and $\varsigma_{3}$ are set to $10 \%$ of the maximum signal amplitude. In the case where waves are not well separated, zones 2 and 3 are merged into a single zone and $\varsigma_{2,3}$ is set to $10 \%$ of the maximum signal amplitude. 


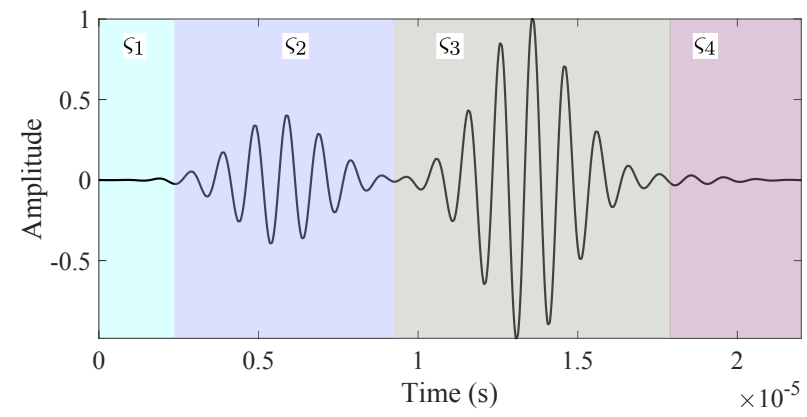

Figure 3: Definitions of zones having a different error $\varsigma_{j}$ on the reference transmitted signal.

\section{A.2 Prior}

The prior probability $\pi_{0}(q)$ encompasses the prior knowledge one has on the parameters, before the new experimental observation (material thickness, physical bounds of certain parameters, etc). The general prior constraints used in this work mostly follow uniform laws, meaning that we attempt an inference as objective as possible [49, restraining ourselves from using informative priors that could help regularize the inference process. The goal is to benchmark the method in a general case where as less a priori as possible is required. In future studies, any prior information on bone samples should be added (such as models based on physical correlations [50], as used in an information theory context by Rus et al. [20]).

Since we aim at the identification of any bone sample, the prior bounds were first taken as large as possible. Uniform prior bounds are given in Table 3 , where a $*$ exponent indicates a measured value for the sample thickness. Note that the angle of incidence $\theta$ (in rad, see Fig. 1) is part of the inferred parameters, due to the uncertainty in the material positioning. The prior probability writes

$$
\pi_{0}(q)=\chi_{\left[q_{\min }, q_{\max }\right]}(q)
$$

where $\chi$ is a uniform density of bounds $q_{\min }$ and $q_{\max }$ (Table 3).

Due to the problem ill-posedness, as noted before in Ref. [30, 36], we found it necessary to constrain the priors on certain parameters in order to regularize the inverse problem, i.e., to remove a multitude of local minima caused by the lack of information contained in a single transmitted signal when attempting to retrieve all the parameters. Since $K_{s}$ and $\rho_{s}$ are tabulated in the literature [46, 51, Table II], we constrained them to case dependent values. Details of these constraints are made explicit in Sec. B for each material. It must be noted that the ill-posedness is mostly related to the elastic parameters $\left(K_{s}, E_{b}\right.$, etc.) rather than to the equivalent fluid parameters $\left(\phi, \alpha_{\infty}, \Lambda\right)$ or the ambient fluid parameters. 


\begin{tabular}{lccccccccccccc}
\hline \hline Parameter & $\theta$ & $L$ & $\phi$ & $\alpha_{\infty}$ & $\Lambda$ & $K_{s}$ & $E_{b}$ & $\xi_{b}$ & $\nu_{b}$ & $\rho_{s}$ & $K_{f}$ & $\mu_{f}$ & $\rho_{f}$ \\
\hline \hline$q_{\min }$ & 0 & $0.9 L^{*}$ & 0.4 & 1.0 & 1 & $\mathrm{CD}$ & 0.1 & 0 & 0.1 & $\mathrm{CD}$ & 2.3 & 0.95 & 0.95 \\
$q_{\max }$ & 0.5 & $1.1 L^{*}$ & 0.99 & 2.0 & 500 & $\mathrm{CD}$ & 20 & 0.5 & 0.45 & $\mathrm{CD}$ & 2.5 & 1.05 & 1.05 \\
\hline \hline
\end{tabular}

Table 3: Prior bounds of the parameters for bone samples (same units as in Table 1). CD: case dependent.

Additional physical constraints It is known [43, Eq. 28] that $\alpha_{B}$ (defined in Eq. 6) follows the relationship $\phi<\alpha_{B}$. This leads to the additional constraint $K_{b}<(1-\phi) K_{s}$, which is enforced by a prior added to the variable $x^{*}=(1-\phi) K_{s}-K_{b}$, as

$$
\pi_{0}\left(x^{*}\right)=\chi_{] 0,+\infty[}\left(x^{*}\right)
$$

\section{B Adaptive Differential Evolution MCMC}

A numerical method is used in order to sample directly from the posterior density of interest $\pi\left(q \mid v^{\mathrm{obs}}\right)$, without having to calculate the denominator on the right hand side of Eq. 13 . The technique used is a variant of the now classical Markov Chain Monte Carlo (MCMC) approach, whose central idea is to sample from unknown distribution, by a random walk across the parameter space that efficiently explores the posterior density, moving preferentially towards regions of high probability density [52]. Doing so, a Markov Chain whose stationary distribution is the posterior density $\pi\left(q \mid v^{\text {obs }}\right)$ is created [53, 54]. This technique has now been well tested on different cases of acoustic porous media identification [32, 35, 36, 47, 55, 56]. The MCMC approach used in this work is the MT-DREAM ${ }_{z s}$ of Laloy et al. [57], shown to be efficient in sampling complex multimodal densities.

In this work, 3 chains are run in parallel, with a multi-try number of 3 . The chains are run for $5 \cdot 10^{5}$ iterations, of which $10 \%$ are discarded as burn-in, to let the chains decorrelate from their initial states. Convergence is checked a posteriori with a Gelman and Rubin [58] criterion and a visual check of the chains. The acceptance ratio of the Metropolis-Hasting algorithm is around $0.2-0.3$ in all cases, which is deemed acceptable. 


\section{Results}

Three different samples, named $M_{1}, M_{2}$ and $M_{3}$, are tested. $M_{1}$ is an alumina ceramic porous (biomaterial) whose properties are relatively well known, and serves as a validation case. $M_{2}$ is a hydroxyapatite sample, a bone substitute [59]. $M_{3}$ is a cancellous bone sample extracted from a human femoral head. The main results are displayed in Sec. $\mathrm{A}$, and a discussion is provided in Sec. B

\section{A Inference of biomaterial and bone samples}

The results are displayed as follows. The posterior marginal densities of each parameter and each pair of parameters are displayed in a matrix form, in Figs. 4668. The shape of the marginal densities gives an information on the sensitivity of an identified parameter, relative to the inference. A flat posterior density function (pdf) is a synonym of a low sensitivity, while a well defined pdf means that information on a parameter was extracted from the experimental data. The presence of multiple peaks in a pdf relates to the non-unicity of the inverse problem solution: given the data and model, both peak values (called modes) have the same likelihood. The joint posterior densities (extra diagonal elements) give an information on the type of correlation between the identified parameters. As such, a very thin ellipse shape denotes either a strong positive or negative correlation between parameters (depending on its orientation). These correlations are not physical ones, but merely represent a possible joint influence of two parameters within the model.

A summary of the results is given in Table 4 with the Maximum A Posteriori (MAP) and a standard deviation relative to the mean. The different fits between the experimental and numerical data are given in Figs. 5779 where CI stands for credibility interval. Credibility intervals are calculated by taking the envelops of Markov Chain realizations (numerical transmitted signals) for input parameters contained in 63\% (resp. 95\%) of the posterior densities. In addition to the time-domain transmitted signals, the attenuation coefficient $A(\omega)$ and the phase velocity $c(\omega)$ are displayed. Their expressions are [60.

$$
\begin{gathered}
A(\omega)=\frac{20}{L} \log _{10}(|\mathcal{T}(\omega)|) / 100, \quad \text { in } \mathrm{dB} / \mathrm{cm}, \\
c(\omega)=\frac{\omega L}{\Delta \varphi(\omega)+\frac{\omega L}{V_{\text {water }}}}, \quad \text { in } \mathrm{m} / \mathrm{s}
\end{gathered}
$$

where $\mathcal{T}(\omega)$ is the transmission coefficient of Eq. 12, $\Delta \varphi(\omega)$ is the unwrapped phase difference between the incident and transmitted signals, and $V_{\text {water }}=1482 \mathrm{~m} / \mathrm{s}$ is the speed of sound in the pore filling fluid (here water). For the cases of materials $M_{1}$ and $M_{2}$, the clear display of the fast and slow waves is evidenced experimentally. This allows the immediate separation of the waves, and a specific calculation of the attenuation 
and phase velocity for each.

The good fit displayed here between numerical and experimental signals (both in time domain and for the attenuation coefficient and phase velocities does not act as a validation of the inferred parameter values, but only as a posterior check. In all cases, the identified values of the incident angle and of the material thickness were correctly identified. Since they are not parameters of interest in this study, they are not shown below for conciseness. However, we note a strong correlation between these parameters and the tortuosity $\alpha_{\infty}$.

Table 4: Result summary for materials $M_{1}, M_{2}$ and $M_{3}$

\begin{tabular}{|c|c|c|c|c|c|c|c|c|c|c|c|c|c|}
\hline & & $\phi$ & $\alpha_{\infty}$ & $\Lambda$ & $\left|K_{s}\right|$ & $\xi_{s}$ & $\left|E_{b}\right|$ & $\xi_{b}$ & $\nu_{b}$ & $\rho_{s}$ & $K_{f}$ & $\mu_{f}$ & $\rho_{f}$ \\
\hline \multirow{2}{*}{ M1 } & MAP & 0.85 & 1.67 & 41.0 & 282 & 0.09 & 14.0 & 0.04 & 0.1 & 4.1 & 2.4 & 0.97 & 0.94 \\
\hline & St.D / mean $(\%)$ & 5.0 & 18.5 & 47.5 & 11.8 & 45.7 & 14.3 & 40.3 & 14.7 & 6.9 & 1.9 & 2.6 & 2.4 \\
\hline \multirow{2}{*}{ M2 } & MAP & 0.5 & 1.05 & 12.1 & 140.0 & 0.09 & 31.4 & 0.1 & 0.39 & 2.7 & 2.38 & 1.0 & 1.0 \\
\hline & St.D / mean $(\%)$ & 9.8 & 2.9 & 5.4 & 16.8 & 42.4 & 19.2 & 14.2 & 16.0 & 6.3 & 2.1 & 5.4 & 4.7 \\
\hline \multirow{2}{*}{ M3 } & MAP & 0.84 & 1.04 & 40.5 & 14.2 & 0.07 & 0.71 & 0.04 & 0.35 & 1.87 & 2.39 & 0.97 & 0.94 \\
\hline & St.D / mean $(\%)$ & 1.0 & 0.96 & 15.0 & 13.2 & 51.5 & 16.4 & 26.8 & 6.3 & 3.0 & 2.0 & 2.0 & 2.4 \\
\hline
\end{tabular}

\section{B Discussion}

A specific analysis of the results is given for each material below.

$\triangleright$ Material $M_{1}$ is an alumina ceramic foam material, which was tested in water with a $500 \mathrm{kHz}$ centered ultrasonic signal. The constructor gave a material porosity of $\approx 0.85$, which is in excellent agreement with the identified value $\phi=0.85 \pm 0.04$. The known density and Young modulus of alumina are $\rho_{s} \approx 3.9 \mathrm{~g} / \mathrm{cm}^{3}$ and $E_{s} \approx 380 \mathrm{GPa}$ [61, Chap. 3]. The prior box-constraints were chosen accordingly by setting $\rho_{s} \in[3.3,4.5] \mathrm{g} / \mathrm{cm}^{3}$ and $K_{s} \in[150,400] \mathrm{GPa}$. The identified value of density $4.1 \pm 0.28 \mathrm{~g} / \mathrm{cm}^{3}$ matches the theoretical one. Using the identified value of the bulk modulus and Eq. 7 with a Poisson's ratio fixed at $\nu_{s}=0.26\left[62\right.$, a Young's modulus of $E_{s} \approx 406 \mathrm{GPa}$ is obtained, quite close to the expected value. Note the high sensitivity of $E_{s}$ relative to $\nu_{s}$ : taking $\nu_{s}=0.275$ would yield $E_{s} \approx 380 \mathrm{GPa}$. A difficulty in identifying the bulk Poisson's ratio $\nu_{b}$ was not expected: the inference seems to favor 


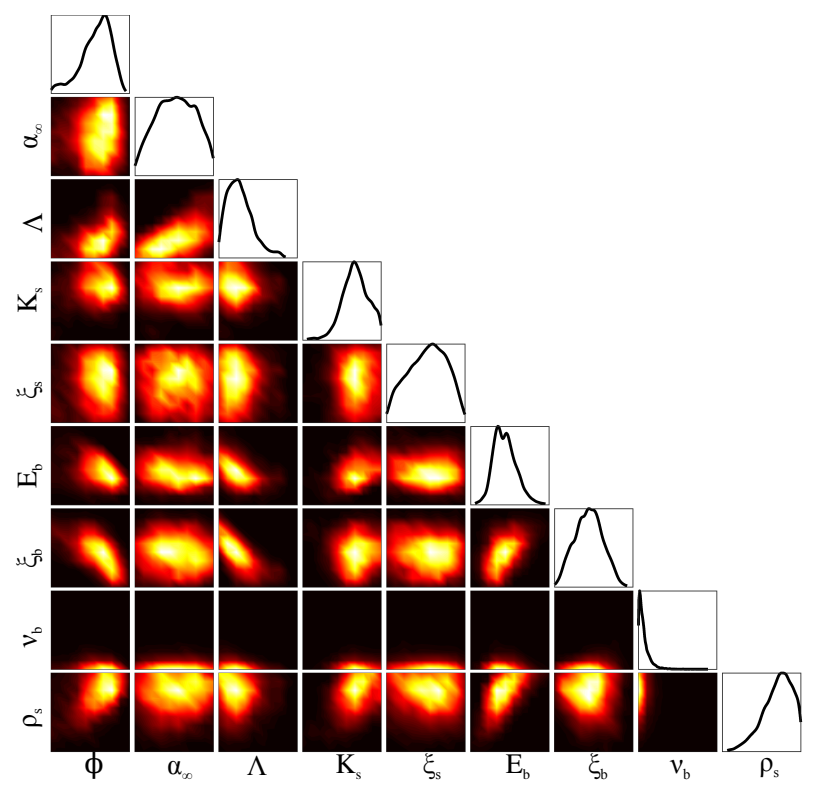

Figure 4: Posterior marginal densities (diagonal elements) and joint densities (extra diagonal elements) for alumina ceramic material $M_{1}$
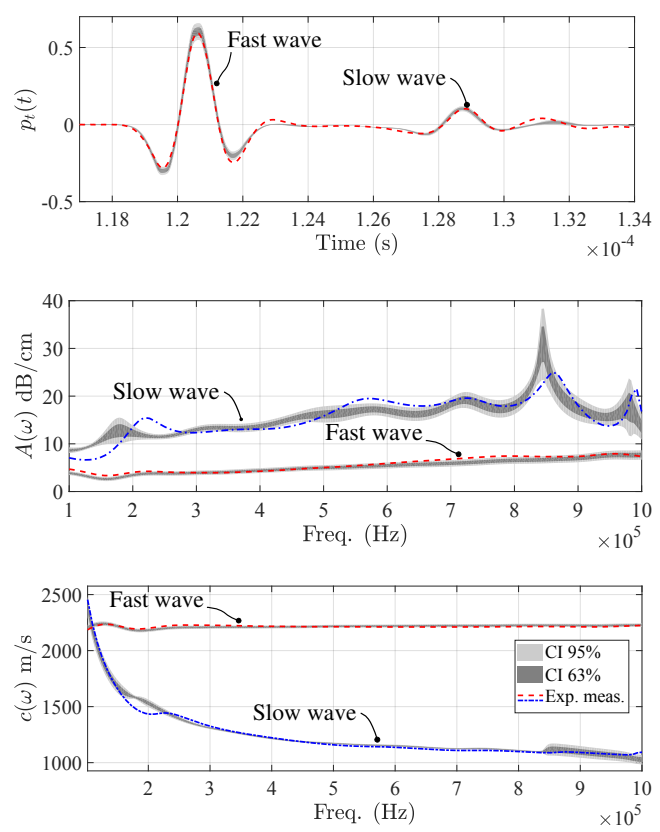

Figure 5: Credibility intervals and experimental ultrasonic measurement for alumina ceramic material $M_{1}$

values close to the lower limit that was given in the prior constraints, see Fig 4 . This problem was absent from other tests. The parameters associated with the ambient fluid (water) are very close to the values known from previous measurements. The relative spread of $\Lambda$ is quite important, compared to the other inference results. This is due to the relatively high value of $\Lambda$, which tends to reduce its sensitivity to the Biot model (the term $2 \delta_{\nu} / \Lambda$ in Eq. 3 becomes small compared to 1 ). Overall, the closeness between identified parameters and independently measured properties gives credit to the identification procedure.

$\triangleright$ Material $M_{2}$ is a bone substitute sample, made of hydroxyapatite, which was tested in water with a $1 \mathrm{MHz}$ centered ultrasonic signal. The prior box-constraints were chosen by setting $\rho_{s} \in[2,4] \mathrm{g} / \mathrm{cm}^{3}$ and $K_{s} \in[80,300] \mathrm{GPa}$. The identified values of porosity $(\phi=0.5)$, Young modulus $\left(\left|E_{b}\right|=31.4 \pm 6 \mathrm{GPa}\right)$ and apparent density, i.e., the $\operatorname{BMD},\left(\rho=(1-\phi) \rho_{s}=1.35 \mathrm{~g} / \mathrm{cm}^{3}\right)$ are quite close to known values $\left(\left|E_{b}\right| \in[35,120] \mathrm{GPa}[63,64]\right)$ and constructor information $\left(\phi \in[0.45,0.85], \rho \in[0.4,1.6] \mathrm{g} / \mathrm{cm}^{3}[65]\right)$. One notes the presence of a bi-modal distribution for the posterior density of the tortuosity, each mode (i.e $\alpha_{\infty}=1.05$ and $\alpha_{\infty}=1.11$ ) having almost the same likelihood. Only the mode related to 


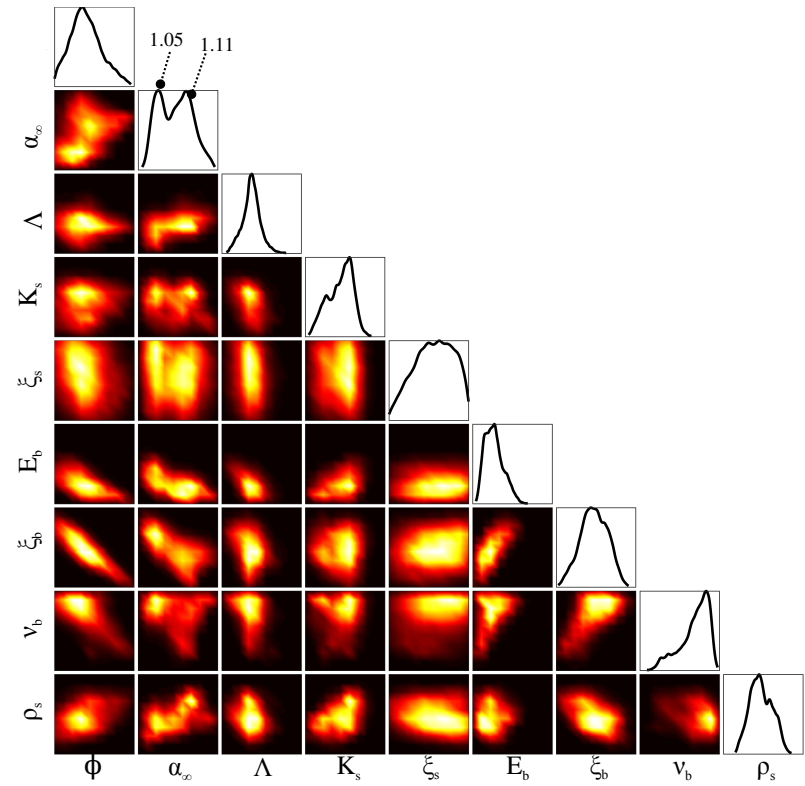

Figure 6: Posterior marginal densities (diagonal elements) and joint densities (extra diagonal elements) for hydroxyapatite material $M_{2}$
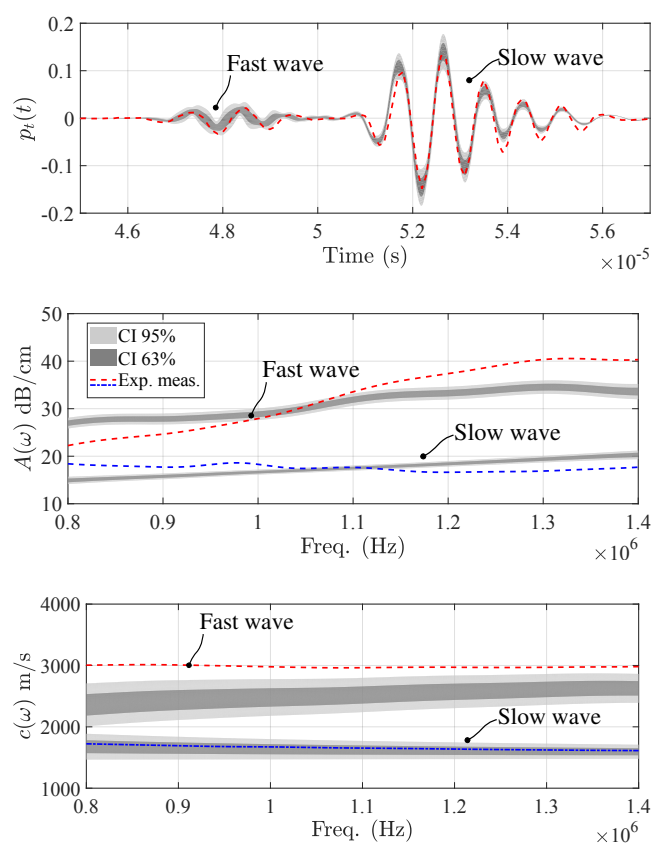

Figure 7: Credibility intervals and experimental ultrasonic measurement for hydroxyapatite material $M_{2}$ 


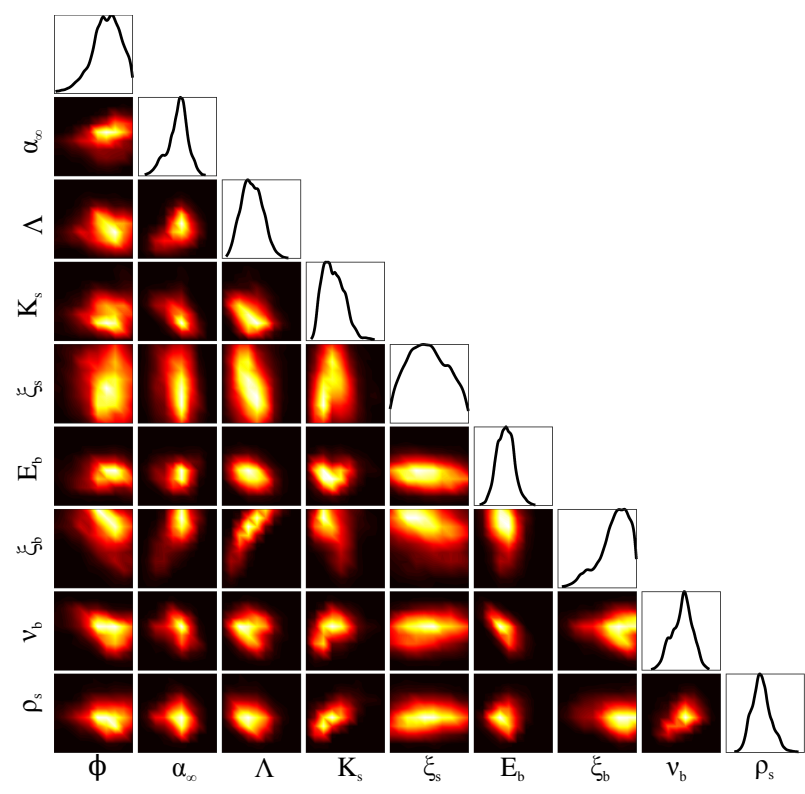

Figure 8: Posterior marginal densities (diagonal elements) and joint densities (extra diagonal elements) for bone sample material $M_{3}$
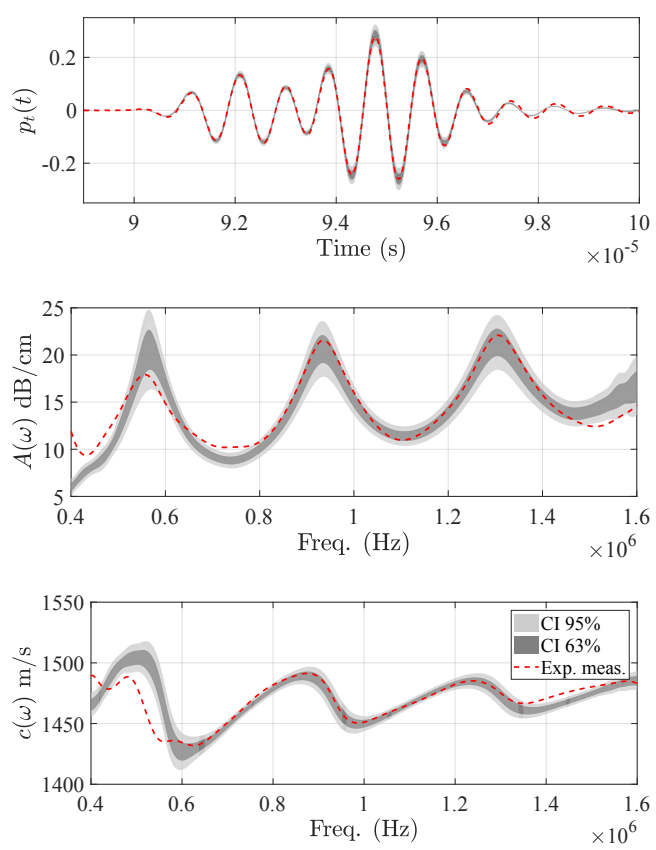

Figure 9: Credibility intervals and experimental ultrasonic measurement for bone sample material $M_{3}$

$\alpha_{\infty}=1.05$ is given in Table 4

$\triangleright$ Material $M_{3}$ is a bone sample extracted from a human femoral head. The material was tested in water with a $1 \mathrm{MHz}$ centered ultrasonic signal. The prior box-constraints were chosen by setting $\rho_{s} \in[1.6,2.2] \mathrm{g} / \mathrm{cm}^{3}$ (see Ref. [66]) and $K_{s} \in[5,30] \mathrm{GPa}$. It was also necessary to further constrain the material density $\rho_{s}$ by adding a prior as

$$
\pi_{0}\left(\rho_{s}\right)=\mathcal{N}(2,0.16)
$$

to avoid the parameter taking either too low or too high a value. A graph is shown in Fig. 10 to compare the present identification results (of Young's modulus versus apparent density) with direct measurements performed by different authors on similar bone samples [67]. The agreement is quite correct, with each contour plot encompassing $10 \%$ of the probabilistic weight of the joint probability density (the external contour encompasses $90 \%$ of the probabilistic weight). In addition, the identified value of solid bulk modulus $\left|K_{s}\right|=14.2 \pm 1.8 \mathrm{GPa}$ is quite close to experimental values obtained by Ashman [68] $\left(E_{s}=13 \pm 1.47 \mathrm{GPa}\right.$, which amounts to $K_{s}=14.4 \pm 1.6 \mathrm{GPa}$, assuming a Poisson's ratio of $\nu_{s}=0.35$ for the solid part of the material). The solid density, identified at $\rho_{s}=1.87 \pm 0.06 \mathrm{~g} / \mathrm{cm}^{3}$, is 


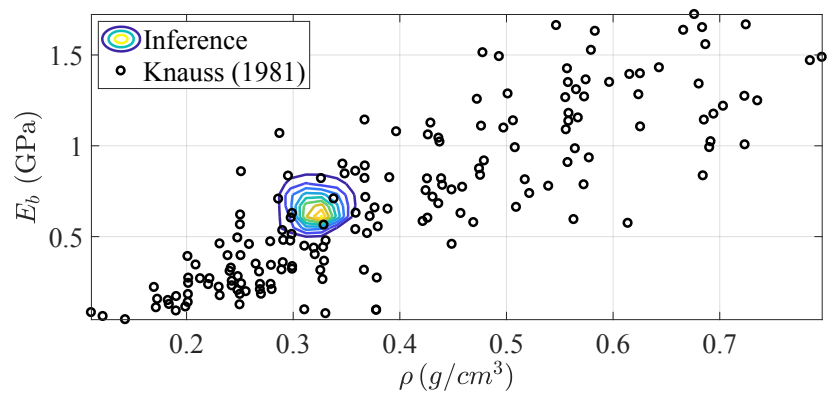

Figure 10: Comparison of inference results with results from Ref. 67]

also well in the range of measured values of $1.76 \pm 0.15 \mathrm{~g} / \mathrm{cm}^{3}$ obtained in Ref. [68, Table 1] by averaging the results of the different human femoral bone samples). We also note that the standard deviation of the identified value of density is well below that for the density prior in Eq. 23, which means that information was indeed gained through the identification process (prior is not too informative). The low value of tortuosity $\alpha_{\infty}=1.04 \pm 0.01$ is coherent with the fact that the test was performed in the bone axial direction, where the trabeculae are mostly straight (for straight pores, the theoretical value is $\alpha_{\infty}=1$ [25, Eq 2.21a with $\left.\left.\theta=0\right]\right)$. The identified porosity $\phi=0.84 \pm 0.01$ is in the range usually cited for trabecular bone samples $(0.88 \pm 0.06$ in Ref. [19]). The porosity is one of the most sensitive parameters in this particular case (lowest relative standard deviation), which means that the transmitted signal yields a significant amount of information on this parameter. Compared with the other inferences, the standard deviations are relatively lower in the case of bone sample $M_{3}$. The sensitivity of each parameter is a complex function of the values of the other parameters, which makes any definitive assessment difficult regarding the increased perceived precision in this particular case.

While realizing this study, multiple possible improvements became apparent.

If possible, the measurement of the reflected wave should be added to the inference process, since it carries information on the microstructure that is different from the information carried by transmitted waves [69]. It has to be noted that while the clear separation of the slow and fast wave is not always present, Biot theory remains valid whether the fast and slow waves are perfectly decoupled or not.

In this work, the microstructural properties of the bone are introduced as model parameters to be identified. Since the inverse problem is ill-posed, adding prior knowledge on these parameters is almost mandatory. Additional prior information could be the knowledge of possible physical correlations between $\phi, \alpha_{\infty}, \Lambda$ in bone samples, to this day unknown. It is thought by the present authors that for a higher porosity $\phi$, the tortuosity $\alpha_{\infty}$ would be lower, while the viscous length $\Lambda$ would be increased. While the 
intrinsic properties of dry bone are different from those of liquid saturated bone [46], approximate values of $\phi, \alpha_{\infty}$ and $\Lambda$ could be obtained as was done in Ref. [55], using the ultrasonic reflected waves from the first interface in air, at different angles of incidence. The first advantage of this technique is that in air, the fluid-structure coupling is reduced. An equivalent fluid approach can then be followed, as in Refs. [36, 47, 55] instead of the full Biot-JKD model, which simplifies the inference procedure and removes most of the problem ill-posedness. Another advantage would be the access to the thermal length $\Lambda^{\prime}$, whose influence cannot be neglected in air-saturated porous media. The thermal length parameter is also related to the pore network micro architecture, and could yield additional insights. For instance, the knowledge of both $\Lambda$ and $\Lambda^{\prime}$ could be used to infer some statistical properties of the pore size distribution (mean and standard deviation), as explained in Ref. [70]. One could also directly use the pore size distribution parameters as model inputs, since they are more easily measured and defined than $\Lambda$ and $\Lambda^{\prime}$ by non-acoustic means. Different physical correlations could also be extracted from previous experimental studies related to bone physical properties, and used to improve the identification process, as done in Ref. [20]. Another point of improvement relates to the anisotropic nature of the bone microstructure. It would certainly be of interest to probe a sample at different incident angles and use all the experimental transmitted signals simultaneously during the inference to obtain the material properties. To represent the anisotropic characteristics of the structure, the tortuosity needs to take into account the wave direction, as done for instance in Ref. [71].

\section{Conclusion}

The identification of the microstructure of bone samples could help in the diagnosis of osteoporosis and other bone related diseases. This article has introduced a general identification method for ceramic and bone samples, based on ultrasonic transmitted signals through the bone porous matrix. A statistical inference strategy has been used to identify simultaneously 9 properties of the porous medium samples, as well as the properties of the fluid saturating the pores (here water). The advantages of this method is that it provides the uncertainty on the model parameters, and a robust way to take into account prior knowledge on the different identified properties.

Three different materials were considered: a ceramic foam of known properties which serves a validation purpose, a bone substitute hydroxyapatite sample and one bone sample extracted from a femoral head. The inverse problem was originally ill-posed, due to a combination of a large number of parameters, an extended search space, the lack of data and the non-linearity of the Biot model used to represent the 
acoustic propagation in bone and biomaterial samples. To limit the problem ill-posedness, prior knowledge

on the material constituent density $\rho_{s}$ and on the material constituent compressibility modulus was used, thus regularizing the solution.

Identification results were in correct agreement with the data obtained in the available literature, and all the material parameters displayed a certain sensitivity relative to the model. The experimental transmitted signals were correctly fitted in all cases, even in the presence of both the slow and fast waves, and for material properties covering a wide range of bio-materials. To continue improving the inference strategy, future work should implement stronger regularizations in the prior density of some of the material properties, by first analyzing a large number of bone samples in air, where the fluid-structure coupling is less important.

\section{Acknowledgment}

This work has been conducted under the ONERA-CNRS collaboration agreement on porous media acoustics. The authors are grateful to Philippe Eyraud for his help with the bibliography work. The authors also wish to thank the anonymous reviewers for their careful reading of the manuscript and their helpful comments.

\section{References}

[1] R. Marcus, D. Feldman, D. Nelson, C. J. Rosen, Fundamentals of osteoporosis, Academic Press, 2009.

[2] J. D. Sibonga, Spaceflight-induced bone loss: is there an osteoporosis risk?, Current osteoporosis reports 11 (2) (2013) 92-98. doi:10.1007/s11914-013-0136-5.

[3] E. S. Orwoll, R. A. Adler, S. Amin, N. Binkley, E. M. Lewiecki, S. M. Petak, S. A. Shapses, M. Sinaki, N. B. Watts, J. D. Sibonga, Skeletal health in long-duration astronauts: nature, assessment, and management recommendations from the nasa bone summit, Journal of bone and mineral research 28 (6) (2013) 1243-1255. doi:10.1002/jbmr.1948.

[4] C. Langton, S. Palmer, R. Porter, The measurement of broadband ultrasonic attenuation in cancellous bone, Engineering in medicine 13 (2) (1984) 89-91. doi:10.1243/EMED_JOUR_1984_013_022_02.

[5] M. McKelvie, S. Palmer, The interaction of ultrasound with cancellous bone, Physics in Medicine \& Biology 36 (10) (1991) 1331. doi:10.1088/0031-9155/36/10/003. 
[6] J. L. Williams, Ultrasonic wave propagation in cancellous and cortical bone: Prediction of some experimental results by biot's theory, The Journal of the Acoustical Society of America 91 (2) (1992) 1106-1112. doi:10.1121/1.402637.

[7] C. F. Njeh, The dependence of ultrasound velocity and attenuation on the material properties of cancellous bone, Ph.D. thesis, Sheffield Hallam University, (1995).

[8] J. Williams, M. Grimm, F. Wehrli, K. Foster, H. Chung, Prediction of frequency and pore size dependent attenuation of ultrasound in trabecular bone using biot's theory, in: Mechanics of Poroelastic Media, Springer, 1996, pp. 263-271. doi:10.1007/978-94-015-8698-6_15.

[9] R. Hodgskinson, C. Njeh, M. Whitehead, C. Langton, The non-linear relationship between bua and porosity in cancellous bone, Physics in Medicine \& Biology 41 (11) (1996) 2411. doi:10.1088/ $0031-9155 / 41 / 11 / 012$

[10] A. Hosokawa, T. Otani, Ultrasonic wave propagation in bovine cancellous bone, The Journal of the Acoustical Society of America 101 (1) (1997) 558-562. doi:10.1121/1.418118.

[11] L. Cardoso, F. Teboul, L. Sedel, C. Oddou, A. Meunier, In vitro acoustic waves propagation in human and bovine cancellous bone, Journal of Bone and Mineral Research 18 (10) (2003) 1803-1812. doi: $10.1359 / \mathrm{jbmr} .2003 .18 \cdot 10.1803$

[12] Z. E. A. Fellah, J. Y. Chapelon, S. Berger, W. Lauriks, C. Depollier, Ultrasonic wave propagation in human cancellous bone: Application of biot theory, The Journal of the Acoustical Society of America 116 (1) (2004) 61-73. doi:10.1121/1.1755239.

[13] P. Laugier, G. Haïat, Bone quantitative ultrasound, Vol. 576, Springer, 2011. doi:10.1007/ 978-94-007-0017-8.

[14] C. Njeh, C. Boivin, C. Langton, The role of ultrasound in the assessment of osteoporosis: a review, Osteoporosis international 7 (1) (1997) 7-22. doi:10.1007/BF01623454.

[15] C.-C. Glüer, I. Q. U. C. Group, Quantitative ultrasound techniques for the assessment of osteoporosis: expert agreement on current status, Journal of Bone and Mineral Research 12 (8) (1997) 1280-1288. doi:10.1359/jbmr.1997.12.8.1280

[16] M. A. Biot, Theory of propagation of elastic waves in a fluid-saturated porous solid. ii. higher frequency range, J. Acoust. Soc. Am. 28 (2) (1956) 179-191. doi:10.1121/1.1908241. 
[17] K. R. Marutyan, M. R. Holland, J. G. Miller, Anomalous negative dispersion in bone can result from the interference of fast and slow waves, The Journal of the Acoustical Society of America 120 (5) (2006) EL55-EL61. doi:10.1121/1.2357187.

[18] E. R. Hughes, T. G. Leighton, P. R. White, G. W. Petley, Investigation of an anisotropic tortuosity in a biot model of ultrasonic propagation in cancellous bone, The Journal of the Acoustical Society of America 121 (1) (2007) 568-574. doi:10.1121/1.2387132.

[19] M. Pakula, F. Padilla, P. Laugier, M. Kaczmarek, Application of biot's theory to ultrasonic characterization of human cancellous bones: determination of structural, material, and mechanical properties, The Journal of the acoustical Society of america 123 (4) (2008) 2415-2423. doi:10.1121/1.2839016.

[20] G. Rus, M. Pakula, Q. Grimal, P. Laugier, Information theory framework to reconstruct biot constants of trabecular bone from ultrasound, in: 2015 6th European Symposium on Ultrasonic Characterization of Bone, 2015, pp. 1-4. doi:10.1109/ESUCB.2015.7169901.

[21] H. Chen, R. P. Gilbert, P. Guyenne, A biot model for the determination of material parameters of cancellous bone from acoustic measurements, Inverse Problems 34 (8) (2018) 085009. doi:10.1088/ 1361-6420/aac520

[22] T. Haire, C. Langton, Biot theory: a review of its application to ultrasound propagation through cancellous bone, Bone 24 (4) (1999) 291 - 295. doi:10.1016/S8756-3282(99)00011-3.

[23] J. Dvorkin, G. Mavko, A. Nur, Squirt flow in fully saturated rocks, Geophysics 60 (1) (1995) 97-107. doi:10.1190/1.1443767,

[24] G. A. Gist, Fluid effects on velocity and attenuation in sandstones, The Journal of the Acoustical Society of America 96 (2) (1994) 1158-1173. doi:10.1121/1.410389.

[25] D. L. Johnson, J. Koplik, R. Dashen, Theory of dynamic permeability and tortuosity in fluid-saturated porous media, J. Fluid Mech. 176 (1987) 379-402. doi:10.1017/S0022112087000727.

[26] N. Sebaa, Propagation des ondes acoustiques dans les milieux poreux saturés: application du modèle de biot à détermination des paramètres de mousses plastiques et de l'os trabéculaire translation; propagation of acoustic waves in porous media: application of the biot model for the determination of plastic foams and trabecular bone properties, Ph.D. thesis, The University of Maine (2006). 
[27] J. Jocker, D. Smeulders, Ultrasonic measurements on poroelastic slabs: Determination of reflection and transmission coefficients and processing for biot input parameters, Ultrasonics 49 (3) (2009) 319-330. doi:10.1016/j.ultras.2008.10.006.

[28] M. Fellah, Z. E. A. Fellah, F. Mitri, E. Ogam, C. Depollier, Transient ultrasound propagation in porous media using biot theory and fractional calculus: Application to human cancellous bone, The Journal of the Acoustical Society of America 133 (4) (2013) 1867-1881. doi:10.1121/1.4792721.

[29] N. Sebaa, Z. E. A. Fellah, M. Fellah, E. Ogam, A. Wirgin, F. Mitri, C. Depollier, W. Lauriks, Ultrasonic characterization of human cancellous bone using the biot theory: Inverse problem, The Journal of the Acoustical Society of America 120 (4) (2006) 1816-1824. doi:10.1121/1.2335420.

[30] E. Ogam, Z. E. A. Fellah, N. Sebaa, J.-P. Groby, Non-ambiguous recovery of biot poroelastic parameters of cellular panels using ultrasonicwaves, Journal of sound and vibration 330 (6) (2011) 1074-1090. doi:10.1016/j.jsv.2010.09.032

[31] J. L. Buchanan, R. P. Gilbert, Y. O. Miao-jung, Recovery of the parameters of cancellous bone by inversion of effective velocities, and transmission and reflection coefficients, Inverse Problems 27 (12) (2011) 125006. doi:10.1088/0266-5611/27/12/125006

[32] M. Niskanen, O. Dazel, J.-P. Groby, A. Duclos, T. Lähivaara, Characterising poroelastic materials in the ultrasonic range-a bayesian approach, arXiv preprint arXiv:1810.01646.

[33] J. Vanhuyse, E. Deckers, S. Jonckheere, B. Pluymers, W. Desmet, Global optimisation methods for poroelastic material characterisation using a clamped sample in a kundt tube setup, Mech. Syst. Sig. Process. 68-69 (2016) $462-478$. doi:10.1016/j.ymssp.2015.06.027.

[34] Y. Champoux, J.-F. Allard, Dynamic tortuosity and bulk modulus in air-saturated porous media, J. Appl. Acoust. 70 (4) (1991) 1975-1979. doi:10.1063/1.349482.

[35] J.-D. Chazot, E. Zhang, J. Antoni, Acoustical and mechanical characterization of poroelastic materials using a bayesian approach, J. Acoust. Soc. Am. 131 (6) (2012) 4584-4595. doi:10.1121/1.3699236.

[36] M. Niskanen, J.-P. Groby, A. Duclos, O. Dazel, J. Le Roux, N. Poulain, T. Huttunen, T. Lähivaara, Deterministic and statistical characterization of rigid frame porous materials from impedance tube measurements, J. Acoust. Soc. Am. 142 (4) (2017) 2407-2418. doi:10.1121/1.5008742. 
[37] C. C. Anderson, A. Q. Bauer, M. R. Holland, M. Pakula, P. Laugier, G. L. Bretthorst, J. G. Miller, Inverse problems in cancellous bone: Estimation of the ultrasonic properties of fast and slow waves using bayesian probability theory, The Journal of the Acoustical Society of America 128 (5) (2010) 2940-2948. doi:10.1121/1.3493441

[38] J. J. Hoffman, A. M. Nelson, M. R. Holland, J. G. Miller, Cancellous bone fast and slow waves obtained with bayesian probability theory correlate with porosity from computed tomography, The Journal of the Acoustical Society of America 132 (3) (2012) 1830-1837. doi:10.1121/1.4739455.

[39] M. A. Biot, Mechanics of deformation and acoustic propagation in porous media, J. Appl. Acoust. 33 (4) (1962) 1482-1498. doi:10.1063/1.1728759

[40] J. Allard, N. Atalla, Propagation of Sound in Porous Media: Modelling Sound Absorbing Materials 2e, John Wiley \& Sons, New York, 2009. doi:10.1002/9780470747339.

[41] L. Gibson, The mechanical behaviour of cancellous bone, Journal of biomechanics 18 (5) (1985) 317-328. doi:10.1016/0021-9290(85)90287-8.

[42] C. Boutin, Rayleigh scattering of acoustic waves in rigid porous media, The Journal of the Acoustical Society of America 122 (4) (2007) 1888-1905. doi:10.1121/1.2756755.

[43] M. Biot, D. Willis, The elastic coefficients of the theory of consolidation, J. appl. Mech 24 (1957) $594-601$.

[44] G. Gautier, L. Kelders, J.-P. Groby, O. Dazel, L. De Ryck, P. Leclaire, Propagation of acoustic waves in a one-dimensional macroscopically inhomogeneous poroelastic material, The Journal of the Acoustical Society of America 130 (3) (2011) 1390-1398. doi:10.1121/1.3605530

[45] O. Dazel, J.-P. Groby, B. Brouard, C. Potel, A stable method to model the acoustic response of multilayered structures, Journal of Applied Physics 113 (8) (2013) 083506. doi:10.1063/1.4790629.

[46] S. B. Lang, Ultrasonic method for measuring elastic coefficients of bone and results on fresh and dried bovine bones, IEEE Transactions on Biomedical Engineering (2) (1970) 101-105. doi:10.1109/TBME. 1970.4502706

[47] R. Roncen, Z. Fellah, E. Piot, F. Simon, E. Ogam, M. Fellah, C. Depollier, Inverse identification of a higher order viscous parameter of rigid porous media in the high frequency domain, The Journal of the Acoustical Society of America 145 (3) (2019) 1629-1639. doi:10.1121/1.5095403. 
[48] R. C. Smith, Uncertainty quantification: theory, implementation, and applications, Vol. 12, Siam, Philadelphia, 2013.

[49] N. Xiang, C. Fackler, Objective bayesian analysis in acoustics, Acoust. Today 11 (2) (2015) 54-61.

[50] Q. Grimal, G. Rus, W. J. Parnell, P. Laugier, A two-parameter model of the effective elastic tensor for cortical bone, Journal of Biomechanics 44 (8) (2011) 1621 - 1625. doi:10.1016/j.jbiomech.2011.03. 006.

[51] J. Y. Rho, R. B. Ashman, C. H. Turner, Young's modulus of trabecular and cortical bone material: ultrasonic and microtensile measurements, Journal of biomechanics 26 (2) (1993) 111-119. doi:10. 1016/0021-9290(93) 90042-D

[52] W. R. Gilks, S. Richardson, D. Spiegelhalter, Markov chain Monte Carlo in practice, Chapman and Hall/CRC, New York, 1995. doi:10.1201/b14835.

[53] N. Metropolis, A. W. Rosenbluth, M. N. Rosenbluth, A. H. Teller, E. Teller, Equation of state calculations by fast computing machines, The journal of chemical physics 21 (6) (1953) 1087-1092. doi:10.1063/1.1699114

[54] W. K. Hastings, Monte carlo sampling methods using markov chains and their applications, Biometrika 57 (1) (1970) 97-109. doi:10.1093/biomet/57.1.97.

[55] R. Roncen, Z. E. A. Fellah, F. Simon, E. Piot, M. Fellah, E. Ogam, C. Depollier, Bayesian inference for the ultrasonic characterization of rigid porous materials using reflected waves by the first interface, The Journal of the Acoustical Society of America 144 (1) (2018) 210-221. doi:10.1121/1.5044423

[56] R. Roncen, Modélisation et identification par inférence bayésienne de matériaux poreux acoustiques en aéronautique (modelling and bayesian inference identification of acoustic porous materials in aeronautics), Ph.D. thesis, Université de Toulouse (2018).

URL https://hal .archives-ouvertes.fr/tel-02004624

[57] E. Laloy, J. A. Vrugt, High-dimensional posterior exploration of hydrologic models using multipletry dream(zs) and high-performance computing, Water Resour. Res. 48 (1) (2012) n/a-n/a, w01526. doi:10.1029/2011WR010608,

[58] A. Gelman, D. B. Rubin, Inference from iterative simulation using multiple sequences, Statistical science 7 (4) (1992) 457-472. doi:10.1214/ss/1177011136. 
[59] Bone substitute materials, http://www.biomet.co.uk/userfiles/files/Biomaterials/Bone\% 20substitute\%20materials.pdf, last accessed: 2019-03-26.

[60] C. Zhang, L. H. Le, R. Zheng, D. Ta, E. Lou, Measurements of ultrasonic phase velocities and attenuation of slow waves in cellular aluminum foams as cancellous bone-mimicking phantoms, The Journal of the Acoustical Society of America 129 (5) (2011) 3317-3326. doi:10.1121/1.3562560.

[61] L. J. Gibson, M. F. Ashby, Cellular Solids: Structure and Properties, 2nd Edition, Cambridge Solid State Science Series, Cambridge University Press, 1997. doi:10.1017/CB09781139878326.

[62] Alumina properties, https://www.azom.com/properties .aspx?ArticleID=105, last accessed: 201903-26.

[63] W. Suchanek, M. Yoshimura, Processing and properties of hydroxyapatite-based biomaterials for use as hard tissue replacement implants, Journal of Materials Research 13 (1) (1998) 94-117. doi:10.1557/ JMR.1998.0015.

[64] V. Orlovskii, V. Komlev, S. Barinov, Hydroxyapatite and hydroxyapatite-based ceramics, Inorganic Materials 38 (10) (2002) 973-984. doi:10.1023/A:1020585800572.

[65] Biomet Deutschland GmbH, Gustav-Krone-Str. 2, D-14167 Berlin, Bone Substitute Materials : Overview, last accessed : 2019/04/05. doi:10.1121/1.1534607.

URL http://www.biomet.co.uk/userfiles/files/Biomaterials/Bone\%20substitute\% 20materials.pdf

[66] T. M. Keaveny, W. C. Hayes, A 20-year perspective on the mechanical properties of trabecular bone, Journal of biomechanical engineering 115 (4B) (1993) 534-542. doi:10.1115/1.2895536

[67] P. Knauss, Material properties and strength behaviour of spongy bone tissue at the coxal human femur.(author's transl), Biomedizinische Technik. Biomedical engineering 26 (9) (1981) 200-210. doi: $10.1515 /$ bmte.1981.26.9.200

[68] R. B. Ashman, J. Y. Rho, Elastic modulus of trabecular bone material, Journal of biomechanics 21 (3) (1988) 177-181. doi:10.1016/0021-9290(88)90167-4.

[69] Z. E. A. Fellah, F. G. Mitri, M. Fellah, E. Ogam, C. Depollier, Ultrasonic characterization of porous absorbing materials: Inverse problem, J. Sound. Vib. 302 (4) (2007) 746-759. doi:10.1016/j.jsv. 2006.12 .007 
[70] K. V. Horoshenkov, J.-P. Groby, O. Dazel, Asymptotic limits of some models for sound propagation in porous media and the assignment of the pore characteristic lengths, The Journal of the Acoustical Society of America 139 (5) (2016) 2463-2474. doi:10.1121/1.4947540

[71] H. Aygün, K. Attenborough, W. Lauriks, P. A. Rubini, C. M. Langton, Wave propagation in stereolithographical (stl) bone replicas at oblique incidence, Applied Acoustics 72 (7) (2011) 458-463. doi: $10.1016 / j$. apacoust. 2011.01 .010 